\title{
Mechanisms for increased risk of diabetes in chronic liver diseases
}

Gianluca Svegliati-Baroni ${ }^{1,2,3}$, Melania Gaggini ${ }^{4}$, Fabrizia Carli ${ }^{4}$, Chiara Barbieri ${ }^{5}$, Monica Cucco $^{1}$, Ramy Youne ${ }^{6}$, Chiara Rosso ${ }^{6}$, Elisabetta Bugianesi ${ }^{6}$, Amalia Gastaldelli $^{4}$.

${ }^{1}$ Department of Clinical and Molecular Sciences, Polytechnic University of Marche, Ancona, Italy ${ }_{2}$ Liver Injury and Transplant Unit, Ospedali Riuniti di Ancona, Ancona, Italy

${ }^{3}$ Obesity Center, Polytechnic University of Marche, Ancona, Italy

${ }^{4}$ Institute of Clinical Physiology, CNR, Pisa

${ }^{5}$ University of Siena, Italy

${ }^{6}$ Division of Gastroenterology and Hepatology, Department of Medical Sciences, University of Turin, Turin, Italy

\section{Running title: Disposition index in chronic liver disease}

\section{Corresponding author:}

Amalia Gastaldelli PhD

Head of Cardiometabolic Risk Unit

Institute of Clinical Physiology, CNR

via Moruzzi 156100 Pisa Italy

email: amalia.gastaldelli@ifc.cnr.it

Abstract 250

Words 4774 (without abstract, figure legends and references)

Figures 4

Table: 2

\section{Authors emails}

Gianluca Svegliati-Baroni g.svegliati@univpm.it

Chiara Barbieri, chiara.barbieri@ifc.cnr.it

Fabrizia Carli, fcarli@ifc.cnr.it

Monica Cucco, monicacucco@alice.it

Ramy Younes ramy.younes@unito.it 
Chiara Rosso, chiara.rosso@unito.it

Melania Gaggini mgaggini@ifc.cnr.it

Elisabetta Bugianesi, elisabetta.bugianesi@ unito.it

Amalia Gastaldelli amalia@ifc.cnr.it

GRAPHICAL ABSTRACT

\section{Risk of type 2 diabetes (T2D) in liver disease}

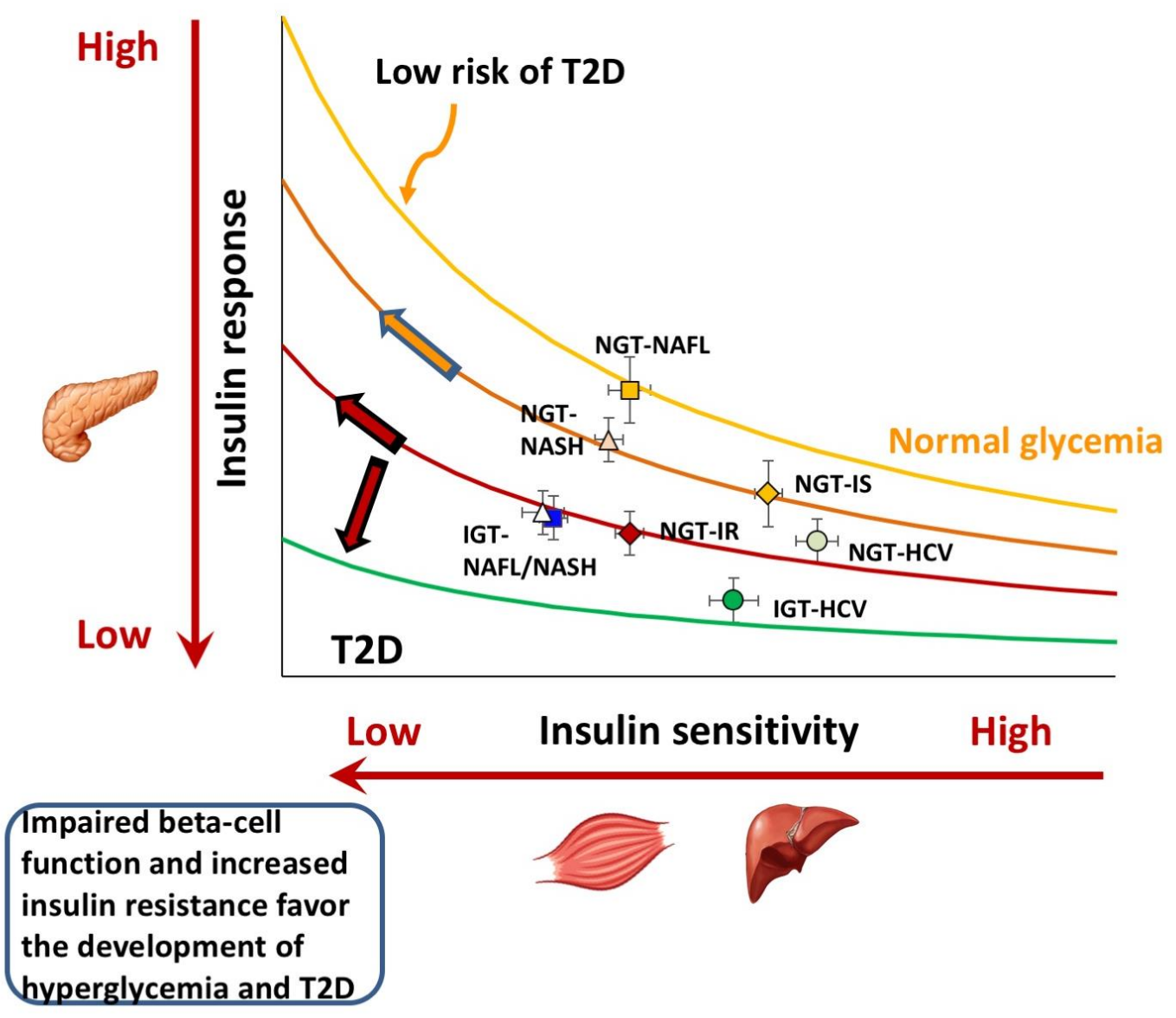


Abstract

Objective. Patients with chronic liver disease (CLD), both non-alcoholic fatty liver disease (NAFLD) and chronic hepatitis $\mathrm{C}(\mathrm{CHC})$, are at high risk of diabetes (T2D), but mechanisms are still unknown. Muscle/liver insulin resistance (IR) and pancreatic dysfunction are the major metabolic defects leading to T2D. However, if the risk of T2D in CLD patients is due to reduced insulin response and/or to IR, and the impact of liver histology has not been investigated.

Design. We studied 220 non-T2D patients with chronic liver disease (129 NAFLD, BMI=27.3 kg/m²; $91 \mathrm{CHC}, \mathrm{BMI}=25.0 \mathrm{~kg} / \mathrm{m}^{2}$ ) that received a 75 -grams OGTT with the measurement of glucose and insulin concentrations for 2 hours, glucose tolerance (NGT vs IGT) and liver biopsy. Results were compared to 26 controls (CT-NGT, BMI $=25.6 \mathrm{~kg} / \mathrm{m}^{2}$ ). We evaluated peripheral insulin sensitivity (OGIS), OGTT-insulin response ( $\triangle \mathrm{AUC}-\mathrm{I} / \Delta \mathrm{AUC}-\mathrm{G})$ and Disposition-Index (DI=OGIS· $\Delta \mathrm{AUC}-$ $\mathrm{I} / \triangle \mathrm{AUC}-\mathrm{G})$ for the risk to develop T2D.

Results. NAFLD had increased muscle-IR (associated to NASH, steatosis and fibrosis), higher than in $\mathrm{CHC}$ or CT-NGT (OGIS=8.9 vs 11.3 and $10.5 \mathrm{ml} / \mathrm{min} \mathrm{kg}$, p<0.0001). In NAFLD OGTT-insulin response ( $\triangle \mathrm{AUC}-\mathrm{I} / \triangle \mathrm{AUC}-\mathrm{G})$ was the highest while it was significantly decreased in CHC (2.2 vs 1.1 and 1.6, NAFLD vs. CHC and CT-NGT, p<0.005). The highest T2D risk (low DI) was observed in CHC-IGT (7.5), CHC-NGT (13.5) and NAFLD-IGT (10.8) vs CT-NGT (14.9, all p<0.0001), but not in NAFL-NGT or NASH-NGT.

Conclusion. We observed an increased T2D risk in NAFLD-IGT, CHC-IGT and CHC-NGT mainly due reduced OGTT-insulin response, while insulin response in NAFLD-NGT compensates the IR thus maintaining normal glycemia. 


\section{SUMMARY BOX}

\section{WHAT IS CURRENT KNOWLEDGE}

- Both NAFLD and HCV infection represents well-known risk factors for Type 2 Diabetes (T2D);

- Pancreatic $\beta$-cell dysfunction and impaired insulin response are the major risk factors in the pathogenesis of T2D since hyperglycemia develops when the amount of insulin secreted by the pancreas is no longer sufficient to overcome the muscle IR;

- It is unknown how alterations of pancreas-liver cross-talk affects T2D development in chronic liver diseases (CLD).

\section{WHAT IS NEW HERE}

- Both NAFLD and CHC patients have alterations of the pancreas-liver cross-talk;

- The major metabolic defect in NAFLD is increased muscle-IR while patients with CHC have impaired pancreatic insulin response after OGTT;

- Increased insulin resistance during OGTT was associated to liver fat accumulation, increased degree of fibrosis and NAS score in patients with NAFLD while no association was found in CHC.

- Disposition Index, DI, was significantly decreased in CHC, particularly if IGT, but not in NAFLNGT or NASH-NGT where the increased insulin response overcome the IR thus maintaining normal glycemia. DI was not associated to the degree of steatosis, neither in NAFLD, nor in CHC and neither to the degree of fibrosis. In NAFLD DI was reduced in those with NAS score greater than 3 . 


\section{Introduction}

In 2015 the global prevalence of type 2 diabetes (T2D) in adults aged 20-79 years was estimated to be $8.8 \%$, but more importantly, almost half (46.5\%) of subjects with T2D did not know to have the disease (1). Chronic liver disease (CLD) is a major risk factor for decreased glucose tolerance and development of type 2 diabetes (T2D) (2-4). Among CLDs non-alcoholic fatty liver disease (NAFLD) is now recognized as an emerging metabolic disease, associated with increased T2D risk and affecting almost $25 \%$ of the world population (5). Similarly, but to a less extent, exposure to hepatitis $\mathrm{C}$ virus (HCV) represents a well-known risk factor for T2D (6). Many studies have shown that all CLDs are associated with insulin resistance (IR) (7-9) although obesity is often not accounted as a covariate despite being an independent risk factor for IR and T2D. Moreover, the impact of reduced $\beta$-cell function and insulin secretion on the risk of T2D in CLD is usually not investigated.

In the pathophysiology of T2D not only IR but also pancreatic $\beta$-cell function and insulin secretion play a major role (10-14). As subjects become insulin resistant (mainly in the periphery), their glucose tolerance is maintained until the amount of insulin secreted by the pancreas is no longer sufficient to overcome the muscle IR $(8,10,11,14)$. Both hepatic and pancreatic dysfunction are major defects that might explain the increased risk of T2D in subjects with liver disease. Alterations in hepatic glucose production and increased postprandial glycemia are early signs of glucose intolerance (14) especially in patients with advanced liver disease even if they have normal fasting glucose concentrations (FPG) (15).

If and how IR and insulin secretion are altered in NAFLD and/or CHC patients is still unknown. The disposition index (DI, calculated from the insulin secretion factored by the insulin resistance) evaluates if the pancreatic insulin secretion is sufficient to overcome peripheral insulin resistance and maintain glycemia within normal ranges $(10,16,17)$. Indeed, a low disposition index has been shown to be an independent marker of development of T2D (17-20). However, to the best of our knowledge the disposition index has not been evaluated in patients with CLD diagnosed by liver biopsy. Moreover, only few studies have investigated $\beta$-cell function and insulin response to a glucose load in subjects with NAFLD or CHC $(15,21-25)$. Despite high hepatic fat accumulation and peripheral IR, $\beta$-cell function was often found unaltered in NAFLD $(21-23,25)$ indicating that most of the patients with CLD are able to compensate their IR by increasing insulin secretion and/or decreasing hepatic insulin clearance. Previously, Grancini et al have evaluated insulin secretion and B-cell function in 160 advanced cirrhotic patients candidates for liver transplantation (the great majority with $\mathrm{HCV}$ ) that underwent OGTT finding that insulin secretion worsens with the worsening of liver disease, and transition from IGT to DM is driven primarily by B-cell dysfunction (15). Narita et al 
have performed OGTT in patients with HCV but IR and ß-cell function were evaluated only according to glucose tolerance and not in relation to the histological severity of the disease (24).

Here we evaluated if and how the type of CLD (NAFLD vs CHC) and severity of liver disease (degree of liver fibrosis) are associated to reduced peripheral insulin sensitivity and insulin response during an OGTT and how the increased risk of T2D of subjects with liver disease (evaluated by the disposition index) is related to alterations of the pancreas-liver cross-talk.

\section{Methods}

\section{Study subjects and protocol}

This is a post-hoc analysis of data of 220 non-diabetic patients with liver disease (NAFLD n=129, $\mathrm{CHC} n=91$ ) that participated to other protocols and that had an OGTT and liver biopsy $(26,27)$. The protocol of data collection was part of the common clinical practice in the hospital units of University of Ancona and Torino. Clinical data have been already published $(26,27)$ while the analysis of $\beta$-cell function is completely new. All subjects were requested to give their informed consent to the use of personal data, analyses and liver biopsy at time of admission. CHC patients were recruited in Ancona between 2003 and 2005 and liver biopsy was performed to evaluate the degree of liver injury before the introduction of Fibroscan in the routine clinical management of these patients in order to define the degree of stage of liver injury and the need of antiviral treatment. $\mathrm{CHC}$ was defined by high transaminase values for more than 6 months and by the presence of serum HCV-RNA in the absence of coinfection with hepatitis B virus (hepatitis B surface antigen and core antibody-positive), autoimmune hepatitis, cholestatic (primary biliary cholangitis, primary sclerosing cholangitis) or genetic (haemochromatosis, a1-antitripsin deficiency, Wilson disease) liver disease. Subjects with pharmacologically treated diabetes or previous antiviral treatment were also excluded. The habitual alcohol intake in the last 6 months was assessed by interviews extended to family members and general practitioners, and patients with alcohol consumption > $40 \mathrm{~g} /$ day were excluded. Other exclusion criteria were the presence of decompensated cirrhosis, the presence of HCC or non-hepatic neoplastic diseases or a low life expectancy due to comorbidities.

NAFLD patients were recruited in Torino and criteria for performing liver biopsy were previously reported $(26,27)$, i.e., chronically elevated aminotransferase levels (alanine aminotransferase (ALT) 1.5 times the upper normal limit for 6 months or more), negative hepatitis B (hepatitis B surface antigen and core antibody) and C (anti-HCV IgG) viral markers, absence of autoimmune hepatitis or coeliac disease, no evidence of genetic, drug-induced or cholestatic liver disease and alcohol consumption (i.e. less than $20 \mathrm{~g} /$ day). Moreover, 26 healthy subjects $\left(\mathrm{BMI}=25.6 \mathrm{~kg} / \mathrm{m}^{2}\right.$ ) previously 
tested with normal glucose tolerance (CT-NGT), without liver disease by ultrasound or, when not available, by fatty liver index, were used as control group.

Liver biopsies were available in all CHC and NAFLD patients and were scored in a blinded manner by two pathologists at the University of Ancona (for CHC patients) and at the University of Turin (for NAFLD patients). Fibrosis was scored according to Metavir (28) for CHC and Kleiner score for NAFLD (29). NASH was diagnosed by the joint presence of steatosis, ballooning and lobular inflammation (30). Patients with a fibrosis score $\geq F$ 2 were defined as high fibrosis (HF).

All subjects received an oral glucose tolerance test (OGTT, 75g) with analysis of the glucose and insulin profiles at 0,30, 60, 90, 120 min after glucose ingestion. Data in CHC and NAFLD patients were compared with those obtained in CT-NGT subjects that were divided according to their response to OGTT as insulin sensitive (NGT-IS) if OGIS $>9.8 \mathrm{ml} / \mathrm{kg} / \mathrm{min}$, vs insulin resistant (NGT-IR) (26). This study was approved by the Ethical Committees of the participating centers (550-586-70-2009 for University of Turin and 205731 for University of Ancona), regulating non-interventional studies. Glucose concentrations were measured with an automated analyser (Beckman Instruments, Fullerton CA, USA; inter-assay coefficient of variation (CV) 4\%) while insulin concentrations were measured by immune-histochemistry assay (AIA-PACK IRI, AIA-1200 system, Tosoh Co., Tokyo, Japan) with intra- and inter-assay CVs for quality control $<7 \%)$ as previously described $(26,27)$.

\section{Calculations}

Subjects were analyzed utilizing the following categories: control subjects were divided according to their response to OGTT as insulin sensitive (NGT-IS) if OGIS $>9.8 \mathrm{ml} / \mathrm{kg} / \mathrm{min}$, vs insulin resistant (NGT-IR); CHC patients were divided according to genotype (G3 vs non-G3) or according to glucose tolerance (CHC-NGT vs CHC-IGT); NAFLD patients were further categorized as NAFL or NASH, or according to glucose tolerance (NAFL-NGT, NASH-NGT, NAFL-IGT, NASH-IGT). Patients were also analyzed according to fibrosis score, ie low (LF) fibrosis (F0-F1) vs high (HF) fibrosis (F2F4). NAS score was calculated as the sum of scores for steatosis, lobular inflammation, and ballooning (30).

Peripheral insulin sensitivity was assessed during OGTT by Oral Glucose Insulin Sensitivity (OGIS) index (31) and at fasting by $\operatorname{HOMA}(32,33)$.

Insulin response to OGTT was assessed by calculating the ratio of incremental area under the curve (AUC) of insulin to glucose $\triangle \mathrm{AUC}-\mathrm{I} / \Delta \mathrm{AUC}-\mathrm{G}$ from 0-120 min (10). The rapid insulin response was assessed as $\triangle \mathrm{AUC}-\mathrm{I} / \triangle \mathrm{AUC}-\mathrm{G}$ from 0-30 min (Insulinogenic index, IGI) (34).

As previously stated, when subjects become insulin resistant (IR), normal glucose tolerance is maintained until amounts of insulin secreted by the pancreas are sufficient to overcome the muscle 
IR $(8,10,11)$. The relationship between insulin sensitivity and insulin secretion is hyperbolic as shown by Bergman et al (35). Thus, by measuring the insulin secretion factored insulin resistance (the so-called disposition index, DI=OGIS x $\Delta$ AUC- $\mathrm{I} / \Delta \mathrm{AUC}-\mathrm{G}$ ) it is possible to evaluate if the pancreatic insulin secretion is sufficient to overcome peripheral insulin resistance and maintain glycemia within normal ranges (10).

\section{Statistical analysis}

For statistical analysis patients were grouped as NAFLD $\left(\mathrm{n}=129, \mathrm{BMI}=27.3 \mathrm{~kg} / \mathrm{m}^{2}\right), \mathrm{CHC}$ genotype $3\left(\mathrm{G} 3, \mathrm{n}=20, \mathrm{BMI}=24.1 \mathrm{~kg} / \mathrm{m}^{2}\right)$ or $\mathrm{CHC}$ non-3 genotype (non-G3, $\mathrm{n}=71, \mathrm{BMI}=25.2 \mathrm{~kg} / \mathrm{m}^{2}$ ) since CHC genotype 3 is known to have a different metabolic profile from $\mathrm{CHC}$ non-3 genotype (7). CT subjects were grouped as insulin sensitive (NGT-IS) or insulin resistant (NGT-IR) according to their response to OGTT (i.e., NGT-IR if Oral Glucose Insulin Sensitivity index OGIS $<9.5 \mathrm{ml} / \mathrm{min} \mathrm{kg}$ ) (26, 27) to better identify differences due to liver disease vs IR.

Data are given as the mean \pm SE. Group differences were analyzed by Student t-test, Mann Whitney test, and $\chi^{2}$ test, for normally distributed, non-normally distributed and non-continuous variables, respectively. Univariate analysis (Spearman correlation coefficient) was used to estimate associations among continuous variables in the whole dataset. A two-tailed $\mathrm{p}<0.05$ was considered statistically significant. Correlation coefficient and p-values were reported in the graphs as well as in the text. 


\section{Results}

Clinical characteristics of the study subjects

We studied 220 non-diabetic patients with liver disease (NAFLD n=129; CHC n=20 for G3 and n=71 for non-G3) and compared to 29 controls without liver disease (Table 1). Control subjects were further divided in 2 groups as insulin sensitive (NGT-IS) or insulin resistant (NGT-IR), according to OGIS $<9.5 \mathrm{ml} / \mathrm{min} \mathrm{kg}$ (Table 1).

CHC patients had a mean BMI $=25.0 \pm 0.4 \mathrm{~kg} / \mathrm{m}^{2}\left(\mathrm{BMI}=24.1 \pm 0.7\right.$ and $25.2 \pm 0.5 \mathrm{~kg} / \mathrm{m}^{2}$ for $\mathrm{G} 3$ and non-G3 respectively) not different from NGT-IS subjects $\left(\mathrm{BMI}=25.9 \pm 0.8 \mathrm{~kg} / \mathrm{m}^{2}\right)$ while NAFLD patients $\left(\mathrm{BMI}=27.3 \pm 0.3 \mathrm{~kg} / \mathrm{m}^{2}\right)$ had a BMI slightly higher than CHC but not controls (Table 1).

AST and ALT were significantly increased in CHC and NAFLD compared to NGT-IS, while GGT was increased only in NAFLD and CHC non-G3 (Table 1).

Lipid profile was similar among the groups, although CHC tended to have lower total cholesterol concentrations. The highest triglyceride (TG) concentrations were observed in the NAFLD group as expected, and in CHC non-G3 (Table 1)

\section{Glucose tolerance in NAFLD vs CHC patients}

All patients underwent a standard OGTT with the measurement of glucose and insulin levels every $30 \mathrm{~min}$ for 2 hours to assess glucose tolerance (Figure 1). The great majority of the patients had normal glucose tolerance (74\% of patients were NGT, Table 1). Based on OGIS index, control subjects were divided in insulin sensitive (NGT-IS), if OGIS $>9.5 \mathrm{ml} / \mathrm{kg} / \mathrm{min}$, vs insulin resistant (NGT-IR). Fasting glucose concentrations were lower in CHC compared to NAFLD, and in CHCG3 they were lower than in controls (Table 1). The great majority of the patients had normal fasting glucose concentrations ( $80 \%$ were NFG, i.e. $<100 \mathrm{mg} / \mathrm{dl}$ ) and a similar distribution was observed in both CHC and NAFLD (impaired fasting glucose, IFG, in 14\% and 24\% respectively, Table 1).

The highest prevalence of impaired glucose tolerant (IGT) subjects was observed in CHC non-G3 (31\%), while in NAFLD it was similar to CHC G3 and control subjects with insulin resistance (26\%, $25 \%$ and $25 \%$ respectively, Table 1). A higher prevalence of IGT was observed also in NAFLD with F2-F4 (Table 2). 


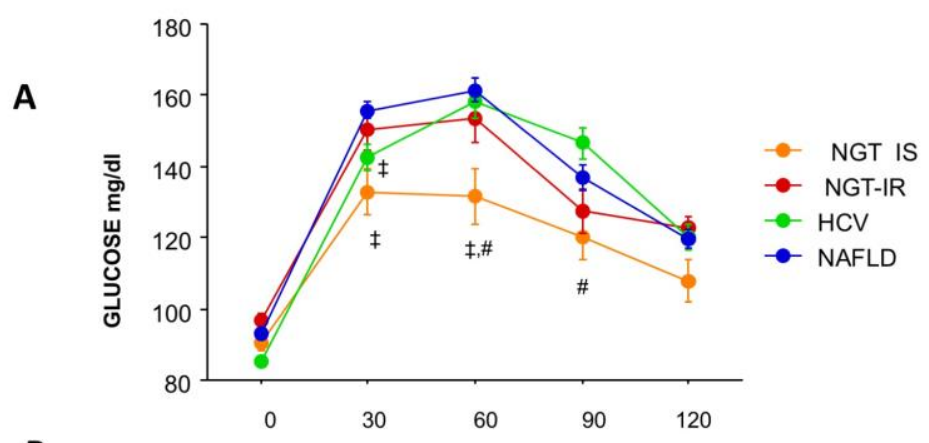

B

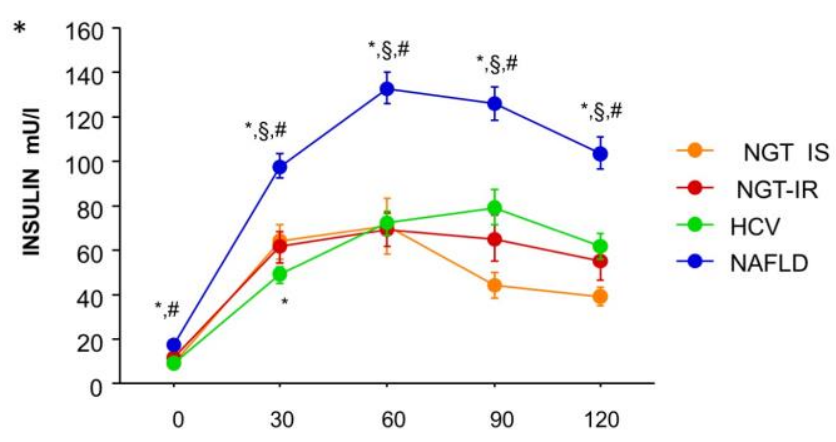

Figure 1. Glucose (Panel A) and Insulin (Panel B) profile during a standard OGTT $(75 \mathrm{~g})$ in controls (insulin sensitive, NGT-IS and insulin resistant, NGT-IR), CHC and NAFLD. Statistical analysis p<0.05: * vs NGT-IS, § vs NGT-IR, \# vs HCV, † vs NAFLD

\section{Insulin sensitivity in NAFLD vs CHC patients}

Fasting insulin resistance was measured by HOMA that was increased only in NAFLD compared to CT-NGT. In CHC patients, HOMA was significantly lower than in NAFLD while there was no difference with CT-NGT despite a lower mean value (Table 1).

Peripheral insulin sensitivity during OGTT was evaluated by OGIS index that is a surrogate measure of glucose clearance during OGTT $(27,31)$. In average, NAFLD had the lowest OGIS $(8.9 \mathrm{ml} / \mathrm{min}$ $\mathrm{kg}$ vs $11.3 \mathrm{ml} / \mathrm{min} \mathrm{kg}$ in CHC and $10.5 \mathrm{in} \mathrm{NGT-IS} \mathrm{ml/min} \mathrm{kg}, \mathrm{p}<0.0001$ ). However, nearly half of the subjects with NAFLD $(\mathrm{n}=55)$ had an OGIS index $>9.8 \mathrm{ml} / \mathrm{min} \mathrm{kg}$. On the other hand, subjects with CHC non-G3 had an OGIS similar to NGT-IS while CHC G3 were even more sensitive than NGT-IS (Table 1). 
When the analysis was performed only in non-obese subjects (i.e. BMI $\leq 30$ ), that comprised the great majority of patients $(85 \%$, i.e. $167 / 200)$, the results were similar.

In NAFLD OGIS was negatively correlated to liver fat in biopsy $(r=-0.31, p=0.0005)$ while no correlation was found in patients with CHC. However, the prevalence of hepatic steatosis was extremely low in patients with $\mathrm{CHC}$ and non G3 genotype, while it was higher in the G3 genotype that has a viral pathogenesis (Table 1).

Since a decrease in OGIS was previously associated to increased liver fibrosis in NAFLD, we also evaluated if the degree of liver fibrosis had an impact on either glucose clearance or insulin response (Table 2, Figure 2). In the entire cohort of subjects with liver disease, patients with advanced fibrosis (F3-F4) had decreased OGIS but, when evaluated separately, only in NAFLD, and not in CHC, reduced OGIS was associated to increased liver fibrosis and this remained significant also after adjusting for BMI and gender (partial $\mathrm{r}=-0.19, \mathrm{p}=0.02$ ), but correlation was lost if further adjusted for age (partial $\mathrm{r}=-0.15, \mathrm{p}=0.08$ ) (Figure 2 panel C). A NAS score greater than 3 (Figure 2 panel E) or presence of NASH were both associated with significantly reduced OGIS.

These data indicate that, although not diabetic, NAFLD patients show decreased peripheral insulin sensitivity compared to controls and this decrease is associated with a higher degree of liver injury. No modifications have been observed in either G3 and non-G3 CHC patients compared to controls. 

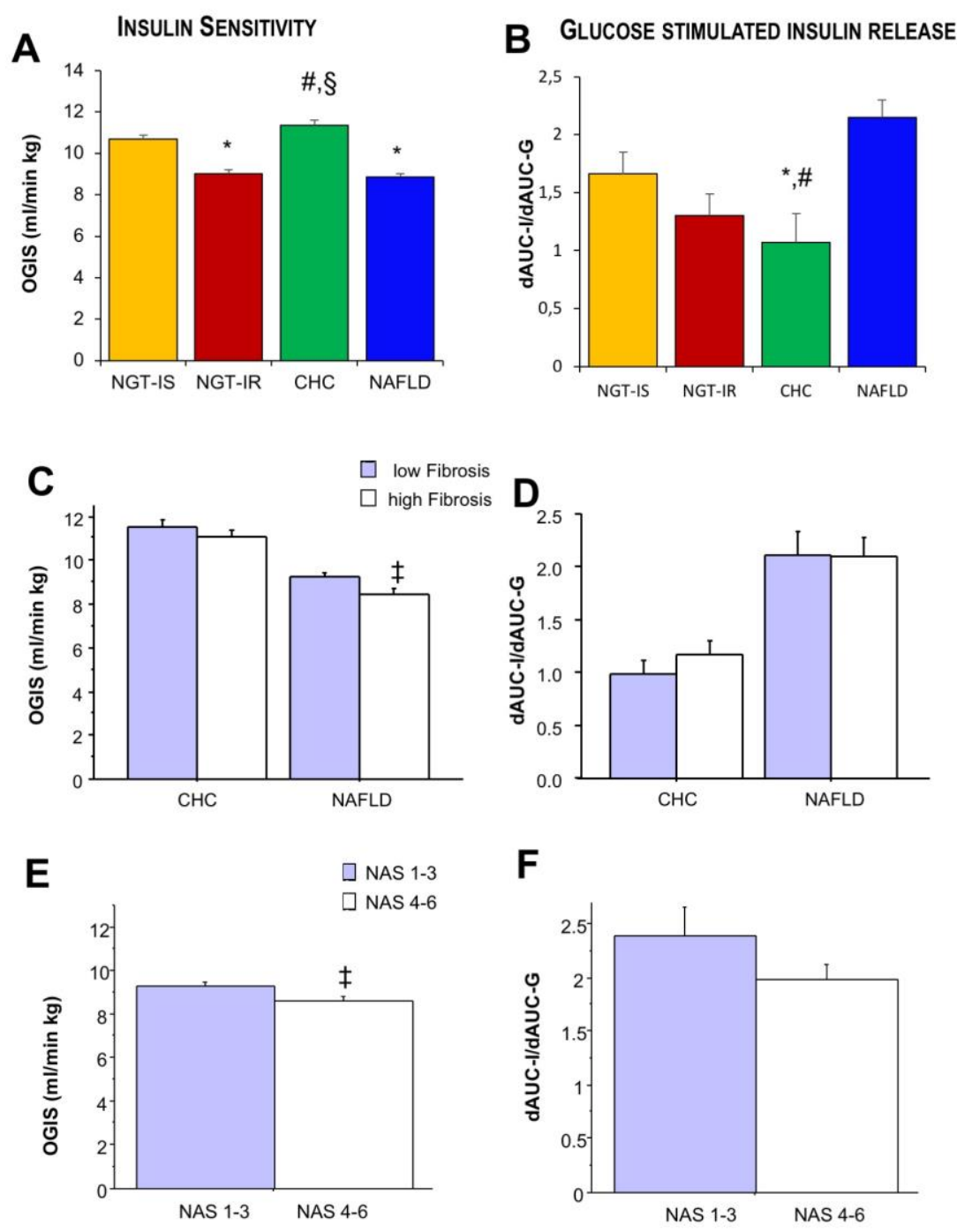

Figure 2. Insulin sensitivity (OGIS index) and insulin secretion calculated as insulin response to OGTT in controls NGT-IS and NGT-IR, CHC and NAFLD (panel A and B); in CHC and NAFLD according to the degree of fibrosis (panel C and D); in NAFLD according to NAS score (panel E and F). The degree of fibrosis was increased with lower OGIS independent of BMI and with increased glucose intolerance in both NAFLD and CHC $(\mathrm{r}=-0.48, \mathrm{p}<0.0001)$. Statistical analysis $\mathrm{p}<0.05$ : * vs NGT-IS, § vs NGT-IR, \# vs NAFLD; $\$$ vs low Fibrosis; $\$$ vs NAS 1-3.

Insulin response to OGTT in NAFLD vs CHC patients

Insulin response to OGTT was assessed by calculating the $\Delta$ AUC-I/ $\triangle A U C-G$ from $0-120 \mathrm{~min}$ (Figure 2 panel B). The highest fasting and OGTT insulin concentrations were observed in NAFLD 
(Figure 1, panel B) despite glucose concentrations were similar to NGT-IR and CHC, indicating a preserved capacity to maintain glucose tolerance by adjusting insulin secretion/hepatic insulin clearance.

In subjects with NAFLD the insulin response to increased glucose concentrations after OGTT was much higher than in $\mathrm{CT}$ while $\triangle \mathrm{AUC}-\mathrm{I} / \triangle \mathrm{AUC}-\mathrm{G}$ was significantly lower in CHC compared to $\mathrm{CT}$ and NAFLD (1.1 vs 2.2 and 1.6, CHC vs NAFLD and NGT-IS, p<0.005) (Figure 1, panel B).

In CHC G3 patients, fasting insulin concentrations were lower than in CT (Table 1) while during OGTT they increased as high as NGT-IR. However, CHC patients, especially those non-G3, had a reduced response in the first 30min, indicating a loss of first phase insulin secretion. In NAFLD, the insulinogenic (IGI) index that reflects the early insulin response was similar to NGT-IS (Table 1) while $\triangle \mathrm{AUC}-\mathrm{I} / \triangle \mathrm{AUC}-\mathrm{G}$ was even higher than in NGT-IS (Figure 2 Panel B).

In NAFLD, but not in CHC, $\triangle \mathrm{AUC}-\mathrm{I} / \triangle \mathrm{AUC}-\mathrm{G}$ was negatively correlated to liver fat in biopsy $(\mathrm{r}=0.20, \mathrm{p}=0.03)$.

No significant association was found between insulin response ( $\triangle \mathrm{AUC}-\mathrm{I} / \Delta \mathrm{AUC}-\mathrm{G})$ and the degree of liver fibrosis in either CHC or NAFLD (Table 2 and Figure 2 panel D), nor with NAS score (Figure 2 panel F) or presence of NASH.

Taken together, these data indicate that insulin levels after OGTT are increased in NAFLD patients to maintain glucose concentrations during OGTT within normal limits, while CHC patients have an insulin profile comparable to NGT-IR.

\section{Disposition index (DI) in NAFLD vs CHC}

In the above analyses we have shown that patients with NAFLD were able to increase insulin response to overcome reduced insulin sensitivity and thus maintain glucose tolerance and do not develop hyperglycemia and T2D.

In each group we evaluated the disposition index (DI calculated as the product of insulin response times insulin resistance sensitivity) according to glucose tolerance status (ie normal glucose tolerance, NGT, vs impaired glucose tolerance, IGT). DI is an index of pancreatic insulin response factored by insulin resistance and inversely associated to increased risk of $\beta$-cell dysfunction and T2D (18, 19, 36). In Panel A of Figure 3 lines are the trajectories of insulin sensitivity and secretion for each given DI and explain why until the subjects remain on the same line they are maintaining a normal glucose tolerance (NGT), i.e., the increased insulin response allows to compensate the reduced insulin sensitivity, and DI is preserved. NGT patients with NAFLD have the same DI, i.e. similar risk to develop T2D, since NAFL-NGT, NASH-NGT and NGT-IS are on similar curve (i.e., similar 
$\mathrm{DI}=24.6 \pm 3.0,18.2 \pm 1.5$ vs $18.0 \pm 3.1$, respectively, $\mathrm{p}=\mathrm{ns}$ ), despite different $\mathrm{IR}$ and insulin secretion (Figure 3, Panel B).

DI was significantly decreased in CHC, particularly if IGT, but not in this group of NAFLD (11.8 \pm vs $18.2 \pm$ vs $16.8 \pm$ in CHC vs NAFLD vs NGT-IS, $\mathrm{p}<0.0001)$ confirming the above observation (Figure 4, Panel A).

DI was not associated to the degree of steatosis, neither in NAFLD, nor in CHC and neither the degree of fibrosis was associated with DI (Figure 4 panel B), but DI was instead decreased with increased glucose intolerance in both NAFLD and CHC (Figure 3 panel B). NAFLD patients with increased NAS score and/or presence of NASH had lower DI (Figure 4 panel C and D).

A
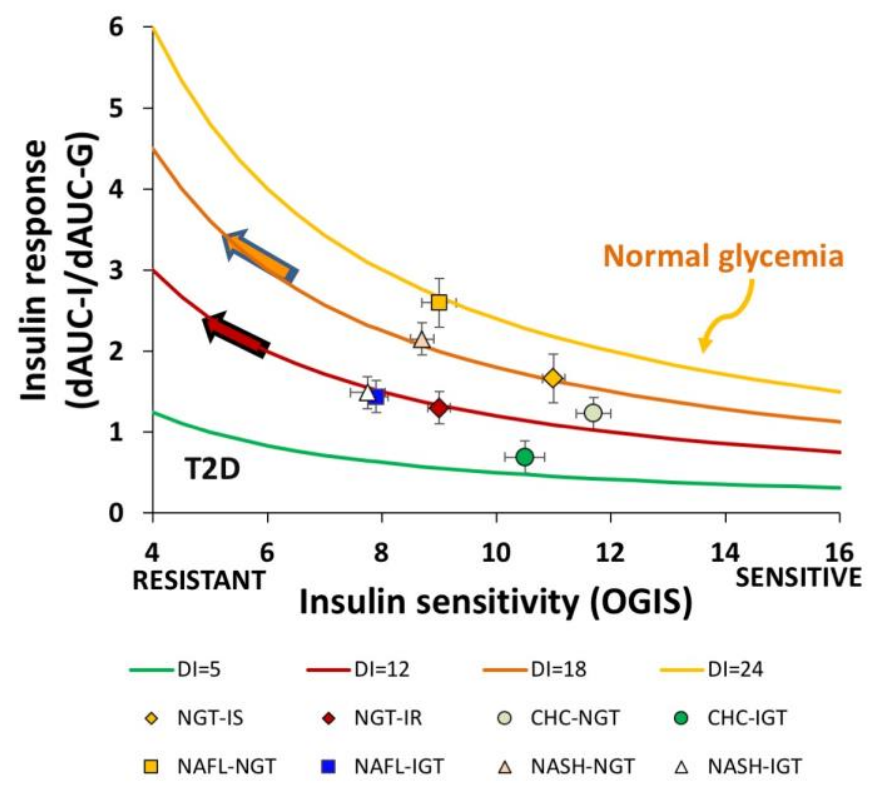

B

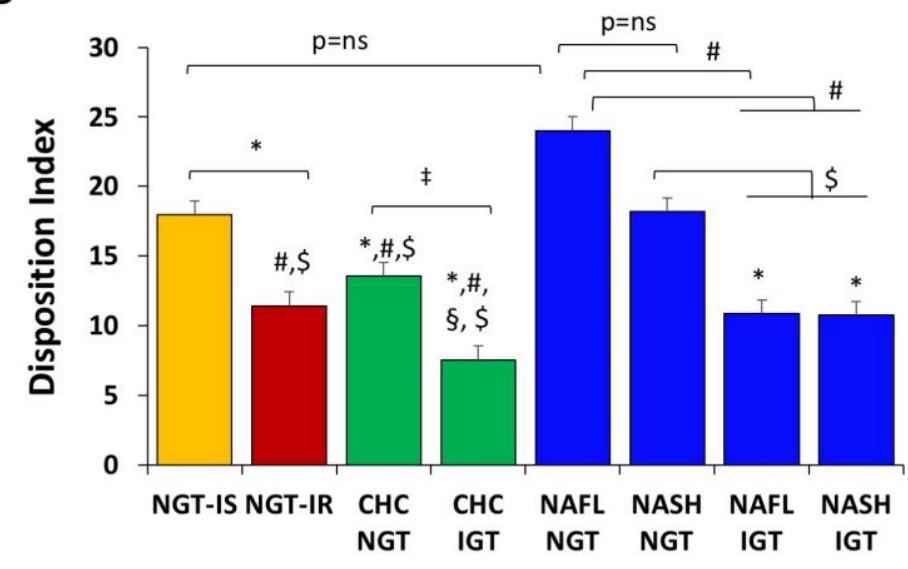

Figure 3. Panel A. Disposition index (DI) trajectories in subjects with or without liver disease. A low DI is a sign of increased risk of type 2 diabetes. In this cohorts, insulin sensitive control subjects 
(NGT-IS), NAFL-NGT and NASH-NGT were on similar curves, indicating that NAFLD patients compensated the lower IS with increased insulin response during OGTT. Subjects with CHC-NGT have reduced DI, similar to NGT-IR, NAFL-IGT and NASH-IGT, while in CHC-IGT the DI was further reduced. Panel B shows the mean values of DI in each group $(* \mathrm{p}<0.05$ vs NGT-IS, $\S \mathrm{p}<0.05$ vs NGT-IR, $\$ \mathrm{p}<0.05$ vs CHC-NGT, \# $\mathrm{p}<0.05$ vs NAFL-NGT $\$ \mathrm{p}<0.05$ vs NASH-NGT following Mann-Whitney comparison among groups).
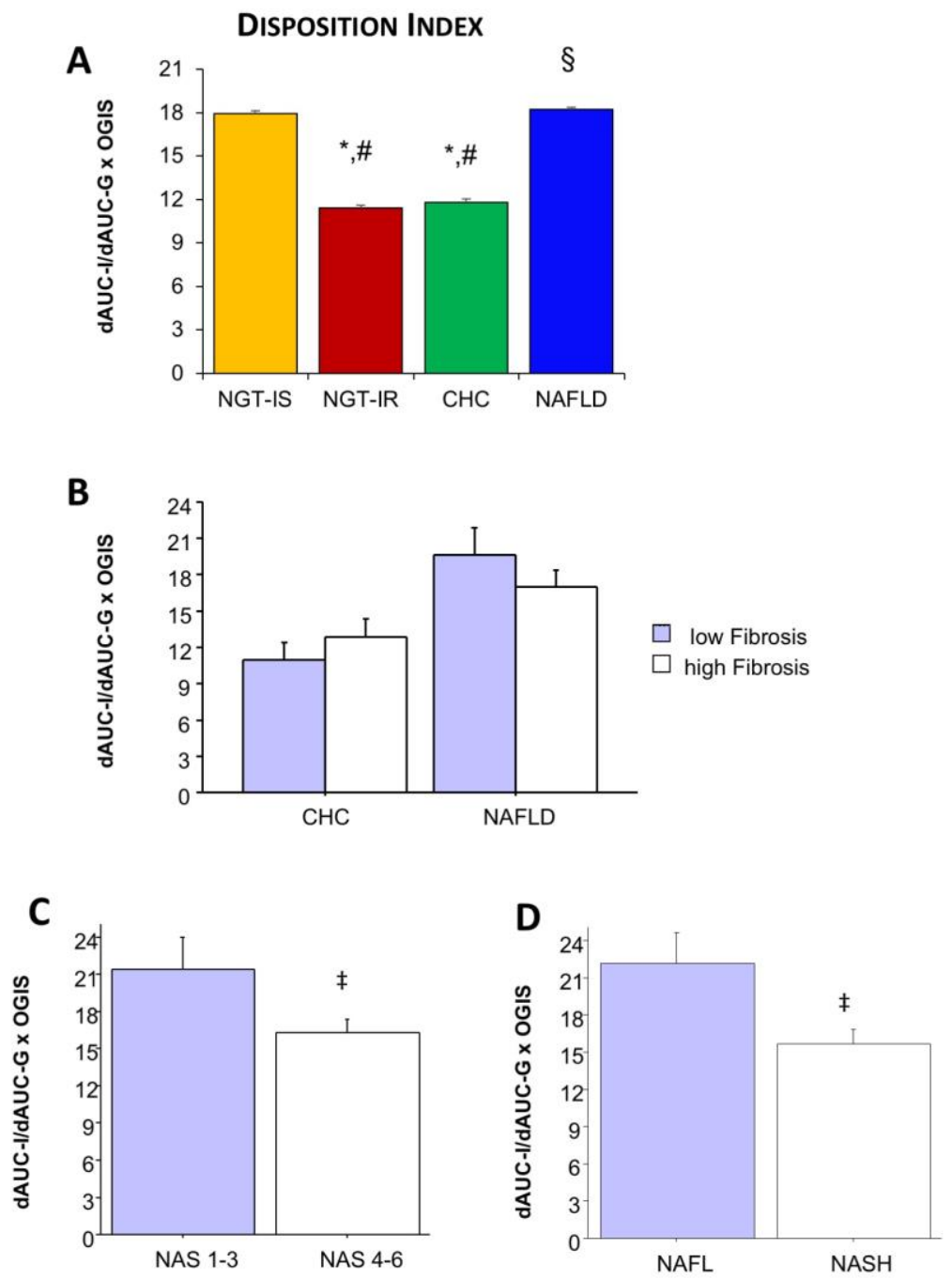

Figure 4. The disposition index (DI) in controls (NGT-IS and NGT-IR), CHC and NAFLD (panel A); in CHC and NAFLD according to the degree of fibrosis (panel B); in NAFLD according to NAS score (panel C); in NAFL vs NASH score (panel D). The degree of fibrosis was not associated with DI. * $\mathrm{p}<0.05$ vs NGT-IS, $\S \mathrm{p}<0.05$ vs NGT-IR, \# $\mathrm{p}<0.05$ vs NAFLD; $\$ \mathrm{p}<0.05$ vs low NAS $1-3$. 


\section{Discussion}

In this study we evaluated data from 220 non-diabetic patients (NAFLD n=129 and CHC n=91) with liver biopsy to verify: a) if and how the type (NAFLD vs CHC) and severity of liver disease (grade of liver inflammation and stage of fibrosis, and presence of NASH) are associated to reduced peripheral insulin sensitivity and/or reduced insulin response during an OGTT; b) how the increased risk of T2D in subjects with liver disease (evaluated by the DI) is related to alterations in the pancreasliver cross-talk. The results of this analysis indicate that, although not diabetic, NAFLD patients had decreased peripheral insulin sensitivity compared to controls and this decrease was associated with presence of NASH and a higher degree of liver injury, while insulin sensitivity of CHC patients was similar to controls. Insulin response to oral glucose load was increased in NAFLD patients compared to both NGT-IS and NGT-IR, but compatible with the degree of IR, so that glucose concentrations during OGTT were within normal limits. On the contrary, CHC patients had a reduced insulin profile comparable to NGT-IR. Considering the risk of T2D (indicated by a low DI) in CLD, the higher risk was observed in CHC and was not associated with the stage of fibrosis, but to the degree of glucose tolerance. This poses the attention on patients that were previously exposed to HCV since this might have altered $B$-cell function thereby favoring the pathogenesis of T2D (37).

Epidemiological studies have shown that both NAFLD and CHC are major risk factors for decreased glucose tolerance and development of type 2 diabetes (T2D) $(2-4,38)$. The prevalence of both diabetes and NAFLD is increasing $(1,5)$ and it has been estimated that almost half of the subjects with diabetes do not know to have the disease since they still have normal fasting glucose concentrations (1). It is recognized that postprandial hyperglycemia occurs much earlier than fasting glycemia (13) since the pancreatic insulin secretion in response to changes in glucose concentrations (e.g. after a meal) is often not sufficient to overcome the peripheral insulin resistance state, thus determining high postprandial glucose levels. Abnormalities in glucose tolerance occur frequently in chronic liver diseases (14), not only in the advanced state and even in patients with normal FPG (15), supporting the importance of performing oral glucose tolerance tests (OGTT). In the pathophysiology of T2D, IR and in particular impaired insulin secretion and B-cell dysfunction are important risk factors, but if they are both altered in NAFLD and CHC patients is still not clear.

The OGTT, with the simultaneous measurement of glucose and insulin concentrations, serves not only to assess glucose tolerance but also insulin secretion (10), insulin resistance in the muscle and liver $(27,31)$ and increased risk to develop type 2 diabetes $(10,19,36)$. Peripheral and hepatic IR are characteristic features of patients with CHC (7) or NAFLD (14), even if they are lean (39). In our recent paper in non-diabetic NAFLD (27) we have shown that, in non-diabetic NAFLD, glucose concentrations during OGTT were similar in obese and non-obese patients independent of the degree 
of liver steatosis, while fibrosis score F2-F4 was associated with increased glucose concentrations during OGTT independent of obesity. On the other hand, insulin concentrations were lower only in non-obese with low steatosis and fibrosis.

We have found that insulin response during OGTT was higher in patients with NAFLD compared to the other groups (Figure 1) in line with other studies $(23,27)$ although appropriate for their glucose tolerance status (14). On the other hand, in patients with $\mathrm{CHC}$ we have found that insulin sensitivity was similar to insulin sensitive of CT-NGT, while glucose-stimulated insulin release was reduced compared to both NAFLD and CT-NGT (Figure 2). Although HCV replicates principally in hepatocytes also other organs might be affected by the virus, like muscle and pancreas $(7,37,40)$. This might explain the alterations in glucose metabolism and tolerance and insulin resistance often observed in CHC patients and their high risk to develop T2D (7, 15). Masini et al have analyzed the pancreatic islets of patients with CHC (37) finding that these patients have both morphological and functional defects, in particular reduced glucose-stimulated insulin release that agrees with our findings (Figure 2, panel B). It has been shown that peripheral insulin sensitivity and glucose metabolism parameters ameliorates after HCV eradication (41-43), but if $\beta$-cell dysfunction improves after Directly Acting Antivirals, or if morphological and functional defects due to virus exposure are permanent, will need further investigation.

The risk to develop T2D was assessed by the evaluation of the insulin secretion/insulin resistance relationship (also named disposition index, DI) $(10,17)$. The disposition index follows a hyperbolic curve that indicates that, until the pancreatic insulin response compensates the reduced insulin sensitivity (see arrows in Figure 4), the subjects preserve their insulin tolerance status, while a decrease in DI indicates an increased risk of T2D $(20,35)$. With the decrease of DI, the patients move on a lower curve increasing their risk to become T2D (i.e. low DI given by a low insulin secretion and/or high insulin resistance).

In this cohort DI was significantly reduced in CHC but not in NAFLD (Figure 4). This was due mainly to the decreased insulin response during OGTT. On the other hand, NAFLD are on the same line as CT despite high insulin resistance (Figure 4). Thus, in this cohort of patients with NAFLD the insulin response is more than adequate to overcome the defect in peripheral insulin resistance thus maintaining glucose tolerance. This is probably due to the fact that most of these patients are nonobese. However, we cannot exclude that the high insulin response in the long term might result in Bcell stress and dysfunction, thus predisposing to diabetes.

We observed that subjects with increased liver fibrosis, in particular NAFLD patients, had lower DI due in part to decreased peripheral insulin sensitivity (OGIS index) but also to peripheral insulin concentrations. We cannot establish if these differences were due to pre-hepatic insulin secretion rates 
or hepatic insulin clearance since we did not measure C-peptide concentrations. In normal conditions the liver clears up to $60 \%$ of the secreted insulin during the first pass, while it does not degrade Cpeptide (44). In liver disease, and/or IR, insulin clearance is reduced in order to have higher insulin concentrations in the periphery $(21,45-47)$. Thus, we cannot establish if increased insulin concentration during OGTT are the result of increased insulin secretion or reduced hepatic clearance or both. However, a recent article has shown that despite NAFLD had a reduced insulin clearance this was dependent on reduced peripheral insulin sensitivity and subcutaneous fat, rather than the degree of liver steatosis (48). Moreover, this is not a limit for the calculation of DI since, as we have shown recently, only peripheral insulin concentrations and not C-peptide are related to insulin sensitivity in a hyperbolic matter and thus insulin and not C-peptide should be used to calculate DI (20).

Another possible limitation is the inclusion in this analysis of mainly non-obese NAFLD patients in order to have a good match for BMI with the CHC cohort. Obesity is often associated to IR and alteration in $\beta$-cell function. However, even in morbid obese subjects, glucose tolerance is often preserved and presence of NAFLD is not associated to impairment in glucose stimulated insulin response (49). Moreover, parameters of $\beta$-cell function such as glucose sensitivity (i.e., dose-response insulin secretion-glucose concentration), first phase insulin secretion, and potentiation, do not appear to be substantially altered by obesity as long as glucose tolerance is maintained (50).

The degree of fibrosis was associated to a reduced OGIS only in NAFLD patients, and it was not associated with DI. On the other hand, DI was decreased with worsening of glucose intolerance in both NAFLD and CHC ( $\mathrm{r}=-0.48, \mathrm{p}<0.0001)$. Why OGIS was associated to hepatic fibrosis only in patients with NAFLD is controversial. The most plausible explanation is that insulin resistance (IR) is the main driver of the hepatic pathological events that finally lead to liver fibrosis, and insulin per se exerts a direct fibrogenetic effect on hepatic stellate cells (HSCs) (51, 52). As a confirmation reduced OGIS was also observed in NAFLD patients with the higher NAS score (steatosis, lobular inflammation and ballooning) that can predispose to fibrosis. On the other hand, although diabetes has been reported to affect almost $15 \%$ of $\mathrm{HCV}$ patients (38), the pathogenesis of $\mathrm{HCV}$-induced liver injury is mostly based on a series of virus-associated events (53). HCV proteins modulate hepatocyte apoptosis and necrosis leading to HSCs and fibrosis. In addition, HCV manipulates the immune system that initially attempts to eradicate the virus, but, in the setting of chronic infection, promotes hepatocyte damage and fibrosis through direct cellular toxicity and the release of inflammatory cytokines. Thus, we can speculate that the role of IR is of minor importance in determining the degree of liver fibrosis in CHC, and this is why liver fibrosis is associated with OGIS in NAFLD and not in CHC. 
In conclusion, the liver plays a central role in metabolic disturbances. Presence of both hepatic and pancreatic dysfunction are major defects that explain the increased risk of T2D in patients with liver disease. Although decreased OGIS was associated to the degree of steatosis, fibrosis and NAS score, patients with NAFLD are able to compensate the increased muscle IR modulating peripheral insulin concentrations. On the other hand, most of CHC patients have an impairment in insulin response to OGTT that increases their risk to develop T2D. While diagnosis of advanced fibrosis by non-invasive tests and treatment are well defined in $\mathrm{HCV}$ patients, these aspects are challenging in NAFLD patients. OGIS measurement after OGTT should be performed in routine clinical practice to identify those NAFLD patients at risk of T2DM and with fibrosis.

\section{Acknowledgments}

\section{Funding}

A.G. and E.B. are recipients of the H2020-2014 EPOS (under grant agreement No. 634413); A.G. is recipient of the grants H2020-MSCA: RISE 2015 MAST4HEALTH (under grant agreement No 691042), H2020-MSCA RISE -2016 mitoFOIE GRAS (under grant agreement No 734719), H2020MSCA ITN-2016 FOIE GRAS (under grant agreement No 722619). 


\section{References}

1. Ogurtsova K, da Rocha Fernandes J D, Huang Y, et al. IDF Diabetes Atlas: Global estimates for the prevalence of diabetes for 2015 and 2040. Diabetes Res Clin Pract 2017; 128: 40-50.

2. Mantovani A, Byrne C D, Bonora E, Targher G. Nonalcoholic Fatty Liver Disease and Risk of Incident Type 2 Diabetes: A Meta-analysis. Diabetes care 2018; 41(2): 372-82.

3. White D L, Ratziu V, El-Serag H B. Hepatitis C infection and risk of diabetes: a systematic review and meta-analysis. J Hepatol 2008; 49(5): 831-44.

4. Kim G, Giannini C, Pierpont B, et al. Longitudinal effects of MRI-measured hepatic steatosis on biomarkers of glucose homeostasis and hepatic apoptosis in obese youth. Diabetes care 2013; 36(1): 130-6.

5. Younossi Z, Anstee Q M, Marietti M, et al. Global burden of NAFLD and NASH: trends, predictions, risk factors and prevention. Nature reviews Gastroenterology \& hepatology 2018; 15(1): 11-20.

6. Negro F, Forton D, Craxi A, Sulkowski M S, Feld J J, Manns M P. Extrahepatic morbidity and mortality of chronic hepatitis C. Gastroenterology 2015; 149(6): 1345-60.

7. Vanni E, Abate M L, Gentilcore E, et al. Sites and mechanisms of insulin resistance in nonobese, nondiabetic patients with chronic hepatitis C. Hepatology 2009; 50(3): 697-706.

8. Gaggini M, Morelli M, Buzzigoli E, DeFronzo R A, Bugianesi E, Gastaldelli A. Nonalcoholic fatty liver disease (NAFLD) and its connection with insulin resistance, dyslipidemia, atherosclerosis and coronary heart disease. Nutrients 2013; 5(5): 1544-60.

9. Mangia A, Schiavone G, Lezzi G, et al. HCV and diabetes mellitus: evidence for a negative association. American Journal Of Gastroenterology 1998; 93: 2363.

10. Gastaldelli A, Ferrannini E, Miyazaki Y, Matsuda M, DeFronzo R A, San Antonio metabolism s. Beta-cell dysfunction and glucose intolerance: results from the San Antonio metabolism (SAM) study. Diabetologia 2004; 47(1): 31-9.

11. DeFronzo R A. Insulin resistance, lipotoxicity, type 2 diabetes and atherosclerosis: the missing links. The Claude Bernard Lecture 2009. Diabetologia 2010; 53(7): 1270-87.

12. Gastaldelli A. Role of beta-cell dysfunction, ectopic fat accumulation and insulin resistance in the pathogenesis of type 2 diabetes mellitus. Diabetes Res Clin Pract 2011; 93 Suppl 1: S60-5.

13. DeFronzo R A, Bonadonna R C, Ferrannini E. Pathogenesis of NIDDM. A balanced overview. Diabetes care 1992; 15(3): 318-68.

14. Gastaldelli A, Cusi K. From NASH to diabetes and from diabetes to NASH: Mechanisms and treatment options. JHEP Reports 2019; 1(4): 312-28.

15. Grancini V, Trombetta M, Lunati M E, et al. Contribution of beta-cell dysfunction and insulin resistance to cirrhosis-associated diabetes: Role of severity of liver disease. J Hepatol 2015; 63(6): 1484-90.

16. Bergman R N, Phillips L S, Cobelli C. Physiologic evaluation of factors controlling glucose tolerance in man: measurement of insulin sensitivity and beta-cell glucose sensitivity from the response to intravenous glucose. J Clin Invest 1981; 68(6): 1456-67.

17. Kahn S E, Cooper M E, Del Prato S. Pathophysiology and treatment of type 2 diabetes: perspectives on the past, present, and future. Lancet 2014; 383(9922): 1068-83.

18. Lorenzo C, Wagenknecht L E, Rewers M J, et al. Disposition index, glucose effectiveness, and conversion to type 2 diabetes: the Insulin Resistance Atherosclerosis Study (IRAS). Diabetes care 2010; 33(9): 2098-103.

19. DeFronzo R A, Tripathy D, Schwenke D C, et al. Prediction of diabetes based on baseline metabolic characteristics in individuals at high risk. Diabetes care 2013; 36(11): 3607-12.

20. DeFronzo R A, Tripathy D, Abdul-Ghani M, Musi N, Gastaldelli A. The disposition index does not reflect beta-cell function in IGT subjects treated with pioglitazone. The Journal of clinical endocrinology and metabolism 2014; 99(10): 3774-81. 
21. Bril F, Lomonaco R, Orsak B, et al. Relationship between disease severity, hyperinsulinemia, and impaired insulin clearance in patients with nonalcoholic steatohepatitis. Hepatology 2014; 59(6): 2178-87.

22. Bedogni G, Gastaldelli A, Manco M, et al. Relationship between fatty liver and glucose metabolism: a cross-sectional study in 571 obese children. Nutr Metab Cardiovasc Dis 2012; 22(2): 120-6.

23. Rijkelijkhuizen J M, Doesburg T, Girman C J, et al. Hepatic fat is not associated with betacell function or postprandial free fatty acid response. Metabolism: clinical and experimental 2009; 58(2): 196-203.

24. Narita R, Abe S, Kihara Y, Akiyama T, Tabaru A, Otsuki M. Insulin resistance and insulin secretion in chronic hepatitis C virus infection. J Hepatol 2004; 41(1): 132-8.

25. Finucane F M, Sharp S J, Hatunic M, et al. Liver fat accumulation is associated with reduced hepatic insulin extraction and beta cell dysfunction in healthy older individuals. Diabetol Metab Syndr 2014; 6(1): 43.

26. Svegliati-Baroni G, Bugianesi E, Bouserhal T, et al. Post-load insulin resistance is an independent predictor of hepatic fibrosis in virus $\mathrm{C}$ chronic hepatitis and in non-alcoholic fatty liver disease. Gut 2007; 56(9): 1296-301.

27. Rosso C, Mezzabotta L, Gaggini M, et al. Peripheral insulin resistance predicts liver damage in nondiabetic subjects with nonalcoholic fatty liver disease. Hepatology 2016; 63(1): 107-16.

28. Bedossa P, Poynard T. An algorithm for the grading of activity in chronic hepatitis $\mathrm{C}$. The METAVIR Cooperative Study Group. Hepatology 1996; 24(2): 289-93.

29. Kleiner D E, Brunt E M, Van Natta M, et al. Design and validation of a histological scoring system for nonalcoholic fatty liver disease. Hepatology 2005; 41(6): 1313-21.

30. EASL, Marchesini G, Day C P, et al. EASL-EASD-EASO Clinical Practice Guidelines for the management of non-alcoholic fatty liver disease. J Hepatol 2016; 64(6): 1388-402.

31. Mari A, Pacini G, Murphy E, Ludvik B, Nolan J J. A model-based method for assessing insulin sensitivity from the oral glucose tolerance test. Diabetes care 2001; 24(3): 539-48.

32. Wallace T M, Levy J C, Matthews D R. Use and abuse of HOMA modeling. Diabetes care 2004; 27(6): 1487-95.

33. Isokuortti E, Zhou Y, Peltonen M, et al. Use of HOMA-IR to diagnose non-alcoholic fatty liver disease: a population-based and inter-laboratory study. Diabetologia 2017; 60(10): 1873-82.

34. Tura A, Kautzky-Willer A, Pacini G. Insulinogenic indices from insulin and C-peptide: comparison of beta-cell function from OGTT and IVGTT. Diabetes Res Clin Pract 2006; 72(3): 298-301.

35. Bergman R N, Ader M, Huecking K, Van Citters G. Accurate assessment of beta-cell function: the hyperbolic correction. Diabetes 2002; 51 Suppl 1: S212-20.

36. Utzschneider K M, Prigeon R L, Faulenbach M V, et al. Oral disposition index predicts the development of future diabetes above and beyond fasting and 2-h glucose levels. Diabetes care 2009; 32(2): 335-41.

37. Masini M, Campani D, Boggi U, et al. Hepatitis $\mathrm{C}$ virus infection and human pancreatic beta-cell dysfunction. Diabetes care 2005; 28(4): 940-1.

38. Younossi Z, Park H, Henry L, Adeyemi A, Stepanova M. Extrahepatic Manifestations of Hepatitis C: A Meta-analysis of Prevalence, Quality of Life, and Economic Burden.

Gastroenterology 2016; 150(7): 1599-608.

39. Bugianesi E, Gastaldelli A, Vanni E, et al. Insulin resistance in non-diabetic patients with non-alcoholic fatty liver disease: sites and mechanisms. Diabetologia 2005; 48(4): 634-42.

40. Cacoub P, Comarmond C, Domont F, Savey L, Desbois A C, Saadoun D. Extrahepatic manifestations of chronic hepatitis C virus infection. Ther Adv Infect Dis 2016; 3(1): 3-14.

41. Gastaldi G, Gomes D, Schneiter P, et al. Treatment with direct-acting antivirals improves peripheral insulin sensitivity in non-diabetic, lean chronic hepatitis C patients. PLoS One 2019; 14(6): e0217751. 
42. Gualerzi A, Bellan M, Smirne C, et al. Improvement of insulin sensitivity in diabetic and non diabetic patients with chronic hepatitis $\mathrm{C}$ treated with direct antiviral agents. PLoS One 2018; 13(12): e0209216.

43. Carnovale C, Pozzi M, Dassano A, et al. The impact of a successful treatment of hepatitis C virus on glyco-metabolic control in diabetic patients: a systematic review and meta-analysis. Acta diabetologica 2019; 56(3): 341-54.

44. Duckworth W C, Bennett R G, Hamel F G. Insulin degradation: progress and potential. Endocr Rev 1998; 19(5): 608-24.

45. Bonnet F, Ducluzeau P H, Gastaldelli A, et al. Liver enzymes are associated with hepatic insulin resistance, insulin secretion, and glucagon concentration in healthy men and women. Diabetes 2011; 60(6): 1660-7.

46. Kotronen A, Juurinen L, Tiikkainen M, Vehkavaara S, Yki-Jarvinen H. Increased liver fat, impaired insulin clearance, and hepatic and adipose tissue insulin resistance in type 2 diabetes. Gastroenterology 2008; 135(1): 122-30.

47. Gastaldelli A, Cusi K, Pettiti M, et al. Relationship between hepatic/visceral fat and hepatic insulin resistance in nondiabetic and type 2 diabetic subjects. Gastroenterology 2007; 133(2): 496506.

48. Utzschneider K M, Kahn S E, Polidori D C. Hepatic Insulin Extraction in NAFLD Is Related to Insulin Resistance Rather Than Liver Fat Content. The Journal of clinical endocrinology and metabolism 2019; 104(5): 1855-65.

49. Coccia F, Testa M, Guarisco G, et al. Insulin resistance, but not insulin response, during oral glucose tolerance test (OGTT) is associated to worse histological outcome in obese NAFLD. Nutrition, Metabolism and Cardiovascular Diseases.

50. Ferrannini E, Camastra S, Gastaldelli A, et al. beta-cell function in obesity: effects of weight loss. Diabetes 2004; 53 Suppl 3: S26-33.

51. Marra F, Svegliati-Baroni G. Lipotoxicity and the gut-liver axis in NASH pathogenesis. J Hepatol 2018; 68(2): 280-95.

52. Svegliati-Baroni G, Ridolfi F, Di Sario A, et al. Insulin and insulin-like growth factor-1 stimulate proliferation and type I collagen accumulation by human hepatic stellate cells: differential effects on signal transduction pathways. Hepatology 1999; 29(6): 1743-51.

53. Mengshol J A, Golden-Mason L, Rosen H R. Mechanisms of Disease: HCV-induced liver injury. Nat Clin Pract Gastroenterol Hepatol 2007; 4(11): 622-34. 
Table 1. Clinical characteristics of study subjects.

\begin{tabular}{|c|c|c|c|c|c|c|}
\hline & $\begin{array}{l}\text { All } \\
\text { subjects }\end{array}$ & NGT-IS & NGT-IR & CHC G3 & CHC non-G3 & NAFLD \\
\hline $\mathbf{n}$ & 246 & 14 & 12 & 20 & 71 & 129 \\
\hline Age (y) & $44 \pm 1$ & $37 \pm 1$ & $39 \pm 2$ & $40 \pm 2 \mathbb{I}$ & $48 \pm 1 * \# \S$ & $43 \pm 19$ \\
\hline Female /Male & $64 / 182$ & $8 / 6$ & $0 / 12$ & $5 / 15$ & $27 / 44$ & $24 / 105$ \\
\hline BMI $\left(\mathrm{kg} / \mathrm{m}^{2}\right)$ & $26.3 \pm 0.2$ & $25.9 \pm 0.8$ & $25.3 \pm 0.7$ & $24.1 \pm 0.7 \S$ & $25.2 \pm 0.5 \S$ & $27.3 \pm 0.3 \mathbb{I}$ \\
\hline Lean/Ow/Obese & $97 / 114 / 35$ & $5 / 8 / 1$ & $6 / 5 / 1$ & $10 / 10 / 0$ & $37 / 29 / 5$ & $39 / 62 / 28$ \\
\hline Non-Obese (\%) & $86 \%$ & $93 \%$ & $92 \%$ & $100 \%$ & $93 \%$ & $78 \%$ II \\
\hline $\begin{array}{l}\text { Presence of } \\
\text { Steatosis }(\%) \neq\end{array}$ & - & - & - & $74 \%$ II & $32 \%$ & $100 \%$ II \\
\hline $\begin{array}{l}\text { Degree of } \\
\text { Steatosis }(\%)\end{array}$ & - & - & - & $27 \pm 69$ & $9 \pm 2 \S$ & $36 \pm 2 \mathbb{I}$ \\
\hline $\begin{array}{l}\text { Fibrosis } \\
\text { F0-F1/F2-F4 }\end{array}$ & - & - & - & $7 / 13$ & $36 / 35$ & $65 / 64$ \\
\hline AST (U/l) & $53 \pm 3$ & $19 \pm 1$ & $20 \pm 3$ & $84 \pm 16^{* \# \S}$ & $62 \pm 4^{*} \# \S$ & $50 \pm 4^{*} \# \mathbb{I}$ \\
\hline ALT (U/l) & $82 \pm 4$ & $15 \pm 1$ & $17 \pm 2$ & $144 \pm 21 * \# \S \rrbracket$ & $98 \pm 6 * \# \S$ & $77 \pm 4 * \#$ II \\
\hline GGT $(\mathrm{U} / \mathrm{l})$ & $87 \pm 7$ & $15 \pm 1$ & $16 \pm 1$ & $72 \pm 15 \S$ & $82 \pm 12 * \#$ & $106 \pm 11 * \#$ \\
\hline Chol (mg/dl) & $191 \pm 3$ & $192 \pm 8$ & $165 \pm 11$ & $164 \pm 8 * \S$ & $182 \pm 4 \S$ & $203 \pm 4 \#$ \#I \\
\hline HDL (mg/dl) & $51 \pm 1$ & $54 \pm 4$ & $46 \pm 3$ & $50 \pm 5$ & $51 \pm 2$ & $52 \pm 1$ \\
\hline TG $(\mathrm{mg} / \mathrm{dl})$ & $113 \pm 5$ & $61 \pm 6$ & $66 \pm 8$ & $72 \pm 6 \S \rrbracket$ & $107 \pm 9 * \S$ & $133 \pm 7 * \# \Phi$ \\
\hline Glucose (mg/dl) & $90 \pm 1$ & $90 \pm 2$ & $97 \pm 2$ & $81 \pm 3^{*} \# \S$ & $86 \pm 2 \# \S$ & $93 \pm 19$ \\
\hline Insulin (mU/l) & $13 \pm 1$ & $9 \pm 1$ & $12 \pm 1$ & $8 \pm 2 \S$ & $10 \pm 1 \S$ & $17 \pm 1 * \pi$ \\
\hline HOMA & $3.1 \pm 0.2$ & $2.1 \pm 0.2$ & $2.8 \pm 0.2$ & $1.7 \pm 0.4 \S$ & $2.1 \pm 0.2 \S$ & $4.0 \pm 0.3 \mathbb{I}$ \\
\hline 2h PG (mg/dl) & $119 \pm 2$ & $108 \pm 6$ & $123 \pm 3$ & $111 \pm 7$ & $123 \pm 4$ & $120 \pm 3$ \\
\hline$\Delta \mathrm{I} / \Delta \mathrm{G} \mathbf{0 - 3 0 \mathrm { min }}$ & $1.2 \pm 0.1$ & $1.4 \pm 0.2$ & $1.0 \pm 0.2$ & $1.4 \pm 0.6 \rrbracket$ & $0.7 \pm 0.1 * \S$ & $1.4 \pm 0.1 \rrbracket$ \\
\hline NFG/IFG & $198 / 48$ & $12 / 2$ & $10 / 2$ & $18 / 2$ & $60 / 11$ & $98 / 31$ \\
\hline NGT/IGT & $185 / 61$ & $14 / 0$ & $12 / 0$ & $15 / 5^{*} \#$ & $49 / 22 * \#$ & $95 / 34 * \#$ \\
\hline
\end{tabular}


tpresence of steatosis at liver biopsy

$* \mathrm{p}<0.05$ vs NGT-IS, \# $\mathrm{p}<0.05$ vs NGT-IR, § $\mathrm{p}<0.05$ vs NAFLD, II $\mathrm{p}<0.05$ vs CHC non-G3, NGT-IS, controls normal glucose tolerant - insulin sensitive; NGT-IR, control normal glucose tolerant - insulin resistant; NFG, normal fasting glucose; IFG, impaired fasting glucose; NGT normal glucose tolerance; IGT impaired glucose tolerance. 
Table 2. Clinical and metabolic characteristics of subjects with liver disease according to the degree of fibrosis.

\begin{tabular}{|c|c|c|c|c|}
\hline & $\begin{array}{l}\text { CHC } \\
\text { F0-F1 }\end{array}$ & $\begin{array}{l}\text { CHC } \\
\text { F2-F4 }\end{array}$ & $\begin{array}{l}\text { NAFLD } \\
\text { F0-F1 }\end{array}$ & $\begin{array}{l}\text { NAFLD } \\
\text { F2-F4 }\end{array}$ \\
\hline $\mathbf{n}$ & 41 & 50 & 65 & 64 \\
\hline Age (y) & $44 \pm 2$ & $48 \pm 2$ & $41 \pm 1$ & $45 \pm 1$ \\
\hline Female /Male & $16 / 27$ & $16 / 32$ & $9 / 56$ & $15 / 49$ \\
\hline BMI $\left(\mathrm{kg} / \mathrm{m}^{2}\right)$ & $25.0 \pm 0.7$ & $25.0 \pm 0.4$ & $26.3 \pm 0.4$ & $28.2 \pm 0.4 \S$ \\
\hline Lean/Ow/Obese & $24 / 15 / 4$ & $25 / 22 / 1$ & $28 / 29 / 8$ & $12 / 32 / 20$ \\
\hline Non-Obese (\%) & $91 \%$ & $98 \%$ & $88 \%$ & $69 \% \S$ \\
\hline AST (U/l) & $57 \pm 6$ & $76 \pm 7 \S$ & $43 \pm 4$ & $57 \pm 6 \S$ \\
\hline ALT (U/l) & $91 \pm 11$ & $121 \pm 9 \S$ & $65 \pm 4$ & $89 \pm 7 \S$ \\
\hline GGT (U/l) & $85 \pm 19$ & $77 \pm 10$ & $80 \pm 9$ & $132 \pm 19$ \\
\hline Chol (mg/dl) & $177 \pm 6$ & $179 \pm 5$ & $200 \pm 6$ & $206 \pm 5$ \\
\hline HDL (mg/dl) & $50 \pm 3$ & $52 \pm 2$ & $52 \pm 2$ & $51 \pm 2$ \\
\hline TG (mg/dl) & $95 \pm 13$ & $103 \pm 8 \S$ & $121 \pm 8$ & $146 \pm 10$ \\
\hline Glucose (mg/dl) & $88 \pm 2$ & $83 \pm 2$ & $92 \pm 1$ & $95 \pm 1$ \\
\hline Insulin (mU/l) & $10 \pm 1$ & $10 \pm 2$ & $17 \pm 1$ & $18 \pm 1$ \\
\hline HOMА & $2.3 \pm 0.4$ & $1.9 \pm 0.2$ & $3.8 \pm 0.4$ & $4.2 \pm 0.4$ \\
\hline 2h PG (mg/dl) & $119 \pm 5$ & $120 \pm 5$ & $113 \pm 4$ & $126 \pm 4 \S$ \\
\hline $\begin{array}{l}\text { AUC-G } \quad 0-120 m i n \\
(g / l)\end{array}$ & $1.61 \pm 0.06$ & $1.68 \pm 0.05$ & $1.59 \pm 0.04$ & $1.77 \pm 0.04 \S$ \\
\hline AUC-I 0-120min (U/l) & $5.9 \pm 0.6$ & $8.0 \pm 0.8$ & $10.9 \pm 0.8$ & $14.1 \pm 1.0 \S$ \\
\hline$\Delta I / \Delta G \quad 0-30 \mathrm{~min}$ & $0.6 \pm 0.1$ & $1.1 \pm 0.3$ & $1.4 \pm 0.1$ & $1.4 \pm 0.1$ \\
\hline$\Delta I / \Delta G 0-120 \mathrm{~min}$ & $1.0 \pm 0.1$ & $1.1 \pm 0.1$ & $2.2 \pm 0.2$ & $2.1 \pm 0.2$ \\
\hline OGIS & $11.4 \pm 0.3$ & $11.3 \pm 0.4$ & $9.3 \pm 0.2$ & $8.4 \pm 0.2 \S$ \\
\hline
\end{tabular}




\begin{tabular}{l|llll} 
Disposition Index & $10.8 \pm 1.3$ & $12.6 \pm 1.5$ & $19.4 \pm 2.1$ & $17.1 \pm 1.3$ \\
NFG/IFG & $33 / 8$ & $45 / 5$ & $50 / 15$ & $48 / 16$ \\
NGT/IGT & $31 / 10$ & $33 / 17$ & $54 / 11$ & $41 / 23 \S$
\end{tabular}

$\S p<0.05$ F2-F4 vs F0-F1 